\title{
VOLATILIZAÇÃO DE AMÔNIA DO SOLO APÓS A APLICAÇÃO DE UREIA CONVENCIONAL OU COM INIBIDOR DE UREASE $^{(1)}$
}

\author{
Francis Alex Tasca ${ }^{(2)}$, Paulo Roberto Ernani ${ }^{(3)}$, Douglas Antonio Rogeri ${ }^{(4)}$, \\ Luciano Colpo Gatiboni ${ }^{(3)} \&$ Paulo Cézar Cassol $^{(3)}$
}

\begin{abstract}
RESUMO
Volatilização de $\mathrm{NH}_{3}$ é a principal reação que diminui a eficiência de utilização pelas plantas do $\mathrm{N}$ proveniente da ureia, quando ela é aplicada sobre a superfície do solo. A fim de minimizar essa perda, produtos têm sido misturados à ureia para inibir temporariamente a ação da urease. Este trabalho objetivou avaliar alternativas de aplicação de um fertilizante com inibidor de urease, visando a diminuir a volatilização de $\mathrm{NH}_{3}$ relativamente à ureia convencional, em algumas condições ambientais e de solo. Foram desenvolvidos quatro experimentos, todos em condições de laboratório, em 2007 e 2008, em Cambissolo Húmico. Os tratamentos variaram em cada estudo e incluíram combinações de níveis de pH do solo (natural; 5,$5 ; 6,3$; e 6,8$)$, umidade do solo $(5,10$ ou $20 \%$ de água) e temperaturas ambientais ( 18 e $\left.35^{\circ} \mathrm{C}\right)$, além de estados físicos (sólido ou líquido) e de métodos de aplicação dos fertilizantes (na superfície ou incorporado ao solo). As unidades experimentais foram constituídas por bandejas plásticas $(23 \times 51 \times 17 \mathrm{~cm})$ com $12 \mathrm{~kg}$ de solo, numa espessura de $15 \mathrm{~cm}$, sobre as quais foram instaladas câmaras coletoras de $\mathrm{NH}_{3}$. A amônia volatilizada foi determinada em várias épocas, nos primeiros 28 dias após a aplicação dos fertilizantes. $O$ pico de volatilização diária de $\mathrm{NH}_{3}$ ocorreu sempre na primeira semana depois da adição dos fertilizantes ao solo, e aconteceu dois a três dias mais tarde para a ureia com inibidor de urease, em relação à ureia convencional. A volatilização de $\mathrm{NH}_{3}$ nem sempre foi maior para a ureia convencional em comparação ao fertilizante contendo inibidor de urease, tampouco para o estado líquido em relação ao granulado. A volatilização de $\mathrm{NH}_{3}$ aumentou com a elevação do $\mathrm{pH}$, da temperatura e da dose aplicada de $\mathrm{N}$ e foi menor nos extremos de umidade (solo com $5 \%$ ou com $20 \%$ de água). Para os fertilizantes aplicados sobre a superficie do solo, a taxa máxima de perda diária foi
\end{abstract}

\footnotetext{
(1) Parte da Dissertação de Mestrado do primeiro autor apresentada à Universidade do Estado de Santa Catarina (UDESC). Recebido para publicação em fevereiro de 2010 e aprovado em dezembro de 2010.

(2) Mestrando do Programa de Pós-Graduação em Manejo do Solo, Centro de Ciências Agroveterinárias, Universidade do Estado de Santa Catarina - UDESC. Av. Luis de Camões 2090, CEP 88520-000 Lages (SC). E-mail: francistasca@yahoo.com.br

(3) Professor do Departamento de Solos, UDESC. Pesquisador do CNPq. E-mail: prernani@cav.udesc.br

(4) Mestrando do Programa de Pós-Graduação em Manejo do Solo, UDESC. E-mail:douglasrogeri@hotmail.com
} 
correspondente a $14 \mathrm{~kg} \mathrm{ha}^{-1}$ de $\mathrm{N}$, e a perda total acumulada variou de 2 a $50 \%$ do N aplicado, dependendo principalmente do estado físico em que o fertilizante foi aplicado, da umidade do solo e da temperatura ambiente. A incorporação dos fertilizantes amídicos ao solo foi a maneira mais eficaz de minimizar as perdas de N por volatilização.

Termos de indexação: temperatura, pH, formas de aplicação de adubos, fertilizantes nitrogenados.

\title{
SUMMARY: AMMONIA VOLATILIZATION FOLLOWING SOIL APPLICATION OF CONVENTIONAL UREA OR UREA WITH UREASE INHIBITOR
}

\begin{abstract}
Ammonia volatilization is an important process of $N$ loss which decreases the use efficiency of $N$ by plants when urea is applied on the soil surface. To overcome this problem, some chemical compounds were mixed with urea to inhibit the urease action. The purpose of this study was to compare applications of an alternative fertilizer with urease inhibitor to reduce ammonia volatilization with conventional urea, under certain environmental and soil conditions. Four experiments were carried out in 2007 and 2008, under laboratory conditions, with samples of a Humic Haplumbrept. The treatments varied according to each experiment in terms of soil conditions, such as $\mathrm{pH}(4.0,5.5,6.3$ and 6.8$)$, soil water content $(5,10$ or $20 \%$ moisture), temperature $\left(18\right.$ or $\left.35^{\circ} \mathrm{C}\right)$, aside from the fertilizer physical state (solid or liquid) and application method (over the surface or soil-incorporated). The experimental units consisted of plastic trays into which $12 \mathrm{~kg}$ of soil (dry basis) were filled in a $15 \mathrm{~cm}$ layer. Ammonia gas traps were installed across the soil surface. Frequent measurements were performed during the first 28 days of soil-fertilizer incubation. The peak of ammonia volatilization from the soil occurred in the first week after the application of traditional urea, and two or three days later for urea with urease inhibitor. Ammonia loss was not always higher from conventional than from treated urea, nor from solid than from liquid fertilizers. Ammonia volatilization increased with increases in soil $\mathrm{pH}$, temperature and $\mathrm{N}$ rate and was lower at the lowest (5\%) and highest $(20 \%)$ soil moisture content. For surface-applied fertilizers, the maximum daily $N$ loss rate was $14 \mathrm{~kg} \mathrm{ha}^{-1}$ and the total cumulative loss ranged from 2 to $50 \%$ of the applied $\mathrm{N}$, depending mainly on the physical state of the fertilizer, temperature and on soil moisture. Soil incorporation of urea fertilizers was the best option to minimize ammonia volatilization in all treatments.
\end{abstract}

Index-terms: temperature, $p H$, fertilizer application forms, nitrogen fertilizers.

\section{INTRODUÇÃO}

A ureia é o fertilizante nitrogenado mais utilizado no Brasil, devido ao seu menor custo por unidade de $\mathrm{N}$ em relação aos demais adubos que contêm esse nutriente. Todavia, quando ela é aplicada na superfície do solo e sem incorporação, podem ocorrer perdas de $\mathrm{N}$ por volatilização de $\mathrm{NH}_{3}$ (Lara-Cabezas et al., 1997; Sangoi et al., 2003; Rochette et al., 2009a).

Após a aplicação ao solo, a ureia $\left[\mathrm{CO}\left(\mathrm{NH}_{2}\right)_{2}\right]$ é hidrolisada pela enzima urease, resultando na formação de carbonato de amônio $\left[\mathrm{CO}\left(\mathrm{NH}_{2}\right)_{2}+2 \mathrm{H}_{2} \mathrm{O}\right.$ $\left.\rightarrow\left(\mathrm{NH}_{4}\right)_{2} \mathrm{CO}_{3}\right]$, que se decompõe rapidamente, originando amônio, bicarbonato e hidroxila $\left[\left(\mathrm{NH}_{4}\right)_{2} \mathrm{CO}_{3}+\mathrm{H}_{2} \mathrm{O} \rightarrow 2 \mathrm{NH}_{4}{ }^{+}+\mathrm{OH}^{-}+\mathrm{HCO}_{3}{ }^{-}\right]$, o que implica elevação do $\mathrm{pH}$ ao redor dos grânulos do fertilizante (Ernani et al., 2001; Rochette et al., 2009b).
Dessa forma, parte do amônio se converte em $\mathrm{NH}_{3}$, que pode se perder na atmosfera, caso a ureia não seja incorporada ao solo (Sangoi et al., 2003; Rochette et al., 2009a). A quantidade de $\mathrm{N}$ volatilizada após a aplicação superficial de ureia ao solo é muito variável e depende de inúmeros fatores, incluindo condições climáticas e atributos relacionados com o solo. Esse fenômeno pode ser pequeno, totalizando de 1 a $15 \%$ (Sangoi et al., 2003; Cantarella et al., 2008; SanzCobena et al., 2008), ou atingir valores extremamente altos - maiores do que $50 \%$ do $\mathrm{N}$ aplicado (LaraCabezas et al., 2000; Sangoi et al., 2003; Rochette et al., 2009a). Baixos teores de matéria orgânica, reduzida capacidade de troca de cátions (Sangoi et al., 2003) e baixa umidade no solo (Liu et al., 2007), assim como altas doses de $\mathrm{N}$ aplicado (Ma et al., 2010), alta temperatura (Clay et al., 1990), valores elevados de pH (Sengik et al., 2001) e maior presença de cobertura 
vegetal (Rochette et al., 2009c), favorecem a volatilização da $\mathrm{NH}_{3}$ a partir da aplicação de ureia sobre a superfície do solo. A incorporação do fertilizante ao solo, por outro lado, diminui drasticamente esse tipo de perda (Trivelin et al., 2002; Sangoi et al., 2003; Rochette et al., 2009a).

Recentemente, alguns compostos químicos, entre eles o NBPT (tiofosfato de N-butiltriamida), têm sido misturados à ureia com o objetivo de diminuir a velocidade de hidrólise da ureia, por meio da inibição da atividade da urease. Resultados experimentais mostram que esses fertilizantes têm diminuído a atividade da urease (Sanz-Cobena et al., 2008) e, com isso, retardado a hidrólise da ureia (Clay et al., 1990) e, por sua vez, o pico de volatilização (Clay et al., 1990; Rochette et al., 2009b), diminuído assim a quantidade de $\mathrm{NH}_{3}$ volatilizada (Clay et al., 1990; Cantarella et al., 2008; Sanz-Cobena et al., 2008), com reflexos positivos no rendimento das culturas (Cantarella \& Marcelino, 2007). A magnitude desses efeitos positivos tem variado muito com as características de solo, de manejo da lavoura e com as condições climáticas que alteram a volatilização de $\mathrm{NH}_{3}$, na época de aplicação do fertilizante e nos primeiros dias subsequentes a essa prática (Cantarella et al., 2008).

Este estudo teve por objetivo avaliar o efeito do $\mathrm{pH}$, da umidade do solo, da temperatura e da dose e forma de aplicação da ureia convencional ou com inibidor de urease na volatilização de $\mathrm{NH}_{3}$, em experimentos desenvolvidos em condições de laboratório.

\section{MATERIAL E MÉTODOS}

Este estudo foi composto por quatro experimentos, todos desenvolvidos no laboratório de Química e Fertilidade do Solo da Universidade do Estado de Santa Catarina, em Lages, SC, nos anos de 2007 e 2008. Em todos eles foram utilizadas amostras de um Cambissolo Húmico álico (Embrapa, 1999), que tinha $50 \mathrm{~g} \mathrm{~kg}^{-1}$ de matéria orgânica, $400 \mathrm{~g} \mathrm{~kg}^{-1}$ de argila e pH em água de 4,0, em condições de vegetação natural. No conjunto dos experimentos, avaliou-se a influência de atributos de solo ( $\mathrm{pH}$ e umidade) e de fatores relacionados com os fertilizantes nitrogenados (formas e doses de aplicação), além da temperatura, na volatilização de $\mathrm{NH}_{3}$. Foi utilizada a ureia convencional e um fertilizante comercial, constituído pela ureia misturada com inibidor da urease (NBPT), ambos com $45 \%$ de N.

Em todos os experimentos, utilizou-se o delineamento experimental completamente casualizado, com quatro repetições. As unidades experimentais foram constituídas por bandejas plásticas com dimensões de $23 \times 51 \times 17 \mathrm{~cm}$ de largura, comprimento e altura, respectivamente. Cada uma delas continha $12 \mathrm{~kg}$ de solo, à exceção do experimento em que foi avaliado o efeito da umidade do solo. A captação da $\mathrm{NH}_{3}$ volatilizada do solo foi feita em câmaras fechadas contendo $\mathrm{H}_{2} \mathrm{SO}_{4}$. Para isso, utilizou-se garrafa plástica transparente de politereftalato de etileno (PET) com capacidade para 2 L e diâmetro basal de $10 \mathrm{~cm}$, que permaneceu vedada na parte superior, sendo a sua base retirada para que os gases nela entrassem após sua introdução ao solo. Dentro da parte mediana dela, foi colocado um tubo plástico de centrífuga de $50 \mathrm{~mL}$, que foi suspenso por um suporte de arame conectado na sua parte superior, fixado pela tampa. Dentro deste tubo colocou-se solução de $\mathrm{H}_{2} \mathrm{SO}_{4}\left(0,3 \mathrm{~mol} \mathrm{~L}^{-1}\right)$, glicerina $(2 \%$, v/v) e duas fitas de papel-filtro $(2 \times 20 \mathrm{~cm})$, com a finalidade de aumentar a superfície de contato da $\mathrm{NH}_{3}$ com o $\mathrm{H}_{2} \mathrm{SO}_{4}$, conforme método descrito por Miyazawa (2007).

A quantidade de $\mathrm{N}-\mathrm{NH}_{3}$ volatilizada foi determinada por arraste de vapor, em aparelho semimicro Kjeldahl (Tedesco et al., 1995). As volatilizações de $\mathrm{NH}_{3}$ foram quantificadas aos $2,4,6$, $8,13,18,23$ e 28 dias após a aplicação dos tratamentos, em cada um dos experimentos. Esses períodos, portanto, constituíram um fator em cada experimento.

Os dados obtidos em cada experimento foram submetidos à análise de variância a $5 \%$. A partir desses resultados, realizou-se a análise de regressão para as variáveis em que houve significância.

No experimento 1, avaliou-se o efeito da temperatura e da forma de aplicação das duas fontes de $\mathrm{N}$ na volatilização de $\mathrm{NH}_{3}$. Os tratamentos resultaram, portanto, da combinação de três fatores: formas de aplicação (sólida na superfície do solo, SS; sólida incorporada, SI; e líquida na superfície do solo, LS) com fertilizantes nitrogenados (ureia, U, e ureia com inibidor de urease, UIU) e duas temperaturas $\left(18\right.$ e $\left.35^{\circ} \mathrm{C}\right)$. Os fertilizantes foram aplicados ao solo em uma área cilíndrica de $78,5 \mathrm{~cm}^{2}$. Por ocasião da aplicação dos fertilizantes na forma sólida e sobre a superfície do solo, fez-se a distribuição uniforme dos grânulos em toda a área abrangida pela base da câmara coletora. Quando se utilizou a forma sólida, os fertilizantes foram incorporados ao solo até a profundidade de $2 \mathrm{~cm}$, de maneira que nenhum grânulo ficasse sobre a superfície do solo. Para aplicação na forma líquida superficial, foram diluídos $16,67 \mathrm{~g}$ de cada fertilizante em água destilada, sendo $20 \mathrm{~mL}$ dessa solução aplicados na área mencionada. Nos tratamentos que não receberam fertilizante líquido, adicionaram-se $20 \mathrm{~mL}$ de água destilada, a fim de padronizar a umidade do solo. Utilizou-se também um tratamento controle, onde não foi aplicado nenhum fertilizante. Em todos os tratamentos, à exceção do controle, foi aplicado o equivalente a $100 \mathrm{~kg} \mathrm{ha}^{-1}$ de N, utilizando-se como base de cálculo da quantidade de adubo aplicada a área superficial da unidade experimental.

No experimento 2 , avaliou-se a influência da acidez do solo na volatilização de $\mathrm{NH}_{3}$ decorrente da aplicação dos dois fertilizantes nitrogenados. Os tratamentos consistiram da combinação das duas fontes 
nitrogenadas (ureia e ureia com inibidor de urease) com quatro níveis de $\mathrm{pH}$ em água. Os valores de $\mathrm{pH}$ estudados foram: 4,0 (natural), 5,5, 6,3 e 6,8. A dose de N utilizada equivaleu a $100 \mathrm{~kg} \mathrm{ha}^{-1}$ de N, utilizandose como base de cálculo a área superficial. As unidades experimentais foram mantidas na temperatura de $18^{\circ} \mathrm{C}$, dentro de câmaras de crescimento vegetal. Os fertilizantes foram aplicados somente sobre a superfície do solo, conforme método descrito para o experimento 1 .

No experimento 3, avaliaram-se as influências de doses de $\mathrm{N}$ e de formas de aplicação dos dois fertilizantes nitrogenados sobre a volatilização de $\mathrm{NH}_{3}$. As doses de $\mathrm{N}$ foram equivalentes a 100 e $190 \mathrm{~kg} \mathrm{ha}^{-1}$. Os fertilizantes foram aplicados sobre a superfície do solo, nas formas sólida e líquida. As formas de aplicação dos fertilizantes no solo seguiram o mesmo método descrito para o experimento 1 .

No experimento 4, avaliou-se a influência da umidade do solo e da forma de aplicação dos fertilizantes nitrogenados na volatilização de $\mathrm{NH}_{3}$. Os tratamentos consistiram de um fatorial envolvendo três teores de água em solo [solo seco ao ar (contendo $5 \%$ de água), 10 e $20 \%$ de água], dois estados físicos do fertilizante (sólido e líquido) e os dois fertilizantes nitrogenados, além de um controle, no qual não houve a aplicação de $\mathrm{N}$. Utilizou-se a dose de $\mathrm{N}$ equivalente a $100 \mathrm{~kg} \mathrm{ha}^{-1}$. Nele, os fertilizantes foram aplicados somente sobre a superfície do solo, da maneira descrita para o experimento 1. Eles foram previamente diluídos em água destilada, de maneira que a quantidade de fertilizante aplicado e a quantidade de água correspondessem à dose e umidades do solo que se desejou estudar.

Nos quatro experimentos avaliados, o pH das amostras de solo de todos os tratamentos foi elevado para 5,5, por meio do uso de uma mistura de carbonato de cálcio + carbonato de magnésio (reagentes analíticos), na relação de 3:1. Após um mês de incubação, o solo foi seco em estufa a $65^{\circ} \mathrm{C}$ por $72 \mathrm{~h}$ e, a seguir, adicionou-se água destilada aos tratamentos, a fim de que o solo atingisse umidade volumétrica de 10 e $20 \%$.

\section{RESULTADOS E DISCUSSÃO}

\section{Experimento 1. Efeito da temperatura e da forma de aplicação dos fertilizantes}

A adição de inibidor de urease à ureia não influenciou a volatilização de $\mathrm{NH}_{3}$, porém retardou o pico de perda máxima. Apesar de não ter havido diferença na volatilização total acumulada entre a ureia convencional e a ureia com inibidor de ureasee, por isso, os gráficos representam a média dos dois fertilizantes (Figura 1) -, a época em que as perdas ocorreram variou entre eles (Figura 2). A ureia proporcionou o pico de volatilização entre o segundo e o quarto dia após a aplicação do adubo, ao passo que no solo onde foi aplicada ureia com inibidor de urease isso ocorreu, em média, até dois dias mais tarde (Figura 2). Outros estudos (Clay et al., 1990; Rochette et al., 2009b) também têm relatado que a adição de inibidores de urease à ureia retarda o pico de volatilização de $\mathrm{NH}_{3}$, que, para a ureia convencional, concentra-se na primeira semana após a aplicação do fertilizante sobre a superfície do solo (Al-kanani et al., 1991; Sengik \& Kiehl, 1995a; Sangoi et al., 2003; Duarte et. al., 2007; Cantarella et al., 2008). Nas avaliações realizadas após 18 dias da aplicação dos fertilizantes ao solo, a perda de $\mathrm{NH}_{3}$ estabilizou e foi baixa em todos os tratamentos.

A perda máxima diária de $\mathrm{NH}_{3}$ aumentou com o acréscimo da temperatura e variou entre os fertilizantes, de acordo com o estado físico, quando eles foram aplicados sobre a superfície do solo (Figura 2). A quantidade máxima diária de $\mathrm{N}$ volatilizada no tratamento com ureia ocorreu quando esse adubo foi aplicado na forma líquida (ULS), na temperatura de

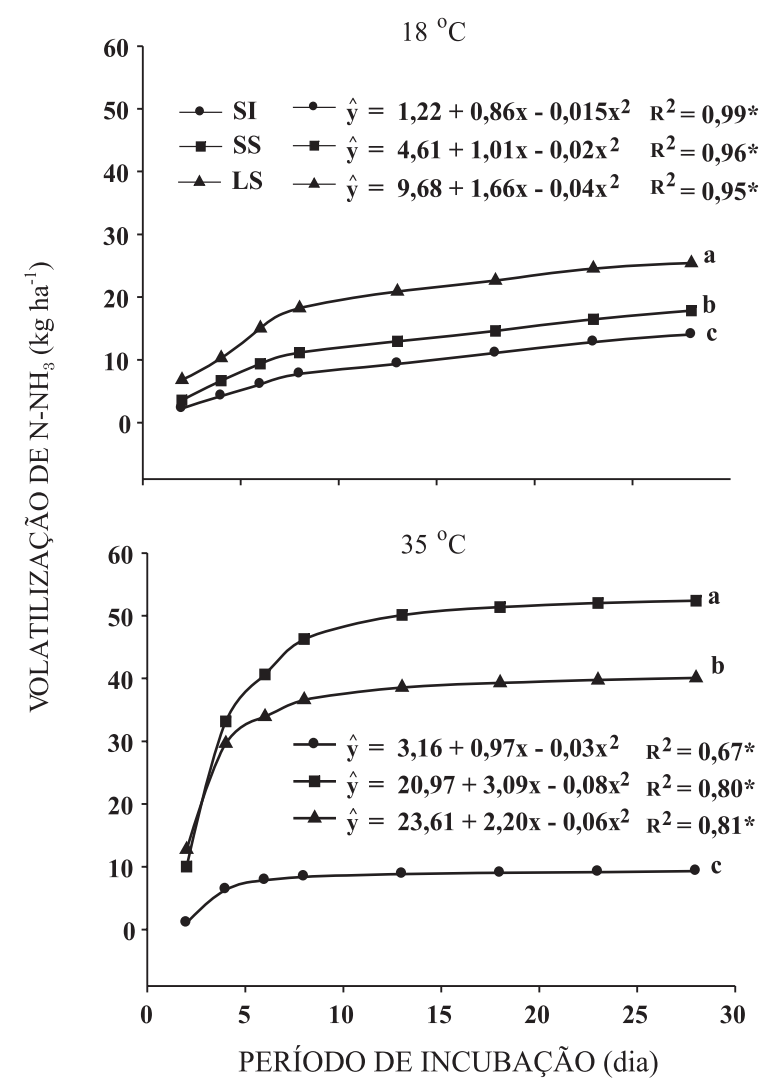

Figura 1. Volatilização acumulada de $\mathrm{NH}_{3}$ do solo ao longo do tempo após a aplicação de ureia convencional ou de ureia com inibidor de urease nas formas sólida, com (SI) ou sem incorporação (SS), ou líquida sobre a superfície (LS), em ambiente mantido a 18 ou a $35^{\circ} \mathrm{C}$. Médias das duas fontes de $\mathrm{N}$. Letras diferentes demonstram que houve diferenças entre as formas de aplicação do fertilizante. * Significativo $(p<0,01)$. 




Figura 2. Volatilização diária de $\mathrm{NH}_{3}$ do solo ao longo do tempo após a aplicação de ureia convencional (U) ou de ureia com inibidor de urease (UIU) nas formas sólida, com (SI) ou sem incorporação (SS), ou líquida sobre a superfície (LS), em ambiente mantido a 18 ou a $35^{\circ} \mathrm{C}$.

$35{ }^{\circ} \mathrm{C}$, equivalendo a $11,1 \mathrm{~kg} \mathrm{ha}^{-1}$; ao passo que, para a ureia com inibidor de urease, aplicada na forma sólida, também na maior temperatura $\left(35^{\circ} \mathrm{C}\right)$, a quantidade máxima diária de $\mathrm{N}$ volatilizada foi de $14,5 \mathrm{~kg} \mathrm{ha}^{-1}$ (Figura 2). As perdas máximas diárias de $\mathrm{N}$ por volatilização de $\mathrm{NH}_{3}$ nos tratamentos com ureia sólida na superfície foram 4,6 vezes menores na temperatura de $18^{\circ} \mathrm{C}$, em relação às observadas na temperatura de $35^{\circ} \mathrm{C}$. Para a ureia com inibidor de urease, o incremento na volatilização ocasionado pelo aumento na temperatura ambiente de 18 para $35^{\circ} \mathrm{C}$ foi de 12 vezes (Figura 2). O efeito do incremento da temperatura na volatilização de $\mathrm{NH}_{3}$ se deve à aceleração na hidrólise da ureia pelo aumento da atividade da urease (Moyo et al., 1989; Clay et al., 1990). O'Connor \& Hendrickson (1987) verificaram que a hidrólise da ureia foi totalmente concluída 1, 4, 6, 7 e 8 dias após a aplicação do fertilizante e associada às temperaturas de $35,25,15,10$ e $5^{\circ} \mathrm{C}$, respectivamente. Ademais, esses autores observaram que, na maior temperatura $\left(35^{\circ} \mathrm{C}\right), 70 \%$ do $\mathrm{N}$ aplicado foi volatilizado em sete dias.

O efeito do aumento da temperatura na volatilização total de $\mathrm{NH}_{3}$ variou com o estado físico em que os fertilizantes foram aplicados sobre a superfície do solo (Figuras 1 e 3). Na temperatura de $18{ }^{\circ} \mathrm{C}$, as maiores perdas acumuladas ocorreram quando os fertilizantes (média da ureia e da ureia com inibidor de urease) foram aplicados por via líquida. Na temperatura de $35^{\circ} \mathrm{C}$, no entanto, a aplicação de ureia sóli- da foi a que proporcionou maior volatilização de $\mathrm{NH}_{3}$ (Figura 1). Quando aplicado sobre a superfície do solo, as perdas totais acumuladas de $\mathrm{N}$ ultrapassaram o equivalente a $50 \mathrm{~kg} \mathrm{ha}{ }^{-1}$, na temperatura de $35^{\circ} \mathrm{C}$, o que representa aproximadamente $50 \%$ do $\mathrm{N}$ aplicado; contudo, na temperatura de $18{ }^{\circ} \mathrm{C}$, menos de $30 \%$ do $\mathrm{N}$ aplicado volatilizou. A maior influência da temperatura na volatilização de $\mathrm{NH}_{3}$ ocorreu nos tratamentos em que os fertilizantes foram aplicados na forma sólida, sobre a superfície do solo (SS); a $35^{\circ} \mathrm{C}$, as perdas excederam em $30 \%$ às ocorridas a $18^{\circ} \mathrm{C}$.

A incorporação dos fertilizantes reduziu drasticamente a volatilização de $\mathrm{NH}_{3}$ (Figura 1). A incorporação da ureia no solo, mesmo em pequenas profundidades, diminui a volatilização (Ernst \& Massey, 1960), em razão de aumentar a chance de o amônio formado ser retido pelas cargas elétricas negativas do solo (Rodrigues \& Kiehl, 1986), à medida que a $\mathrm{NH}_{3}$ difunde para a superfície do solo e encontra valores de $\mathrm{pH}$ mais baixos do que ao redor dos grânulos do fertilizante.

\section{Experimento 2. Efeito da acidez do solo}

A volatilização de $\mathrm{NH}_{3}$ aumentou com o incremento do pH do solo e, à exceção do solo onde a acidez não foi corrigida, foi maior para a ureia convencional do que para a ureia com inibidor de urease (Figura 4). O efeito do $\mathrm{pH}$ na volatilização da $\mathrm{NH}_{3}$ está ligado à hidrólise da ureia (Fan \& Mackenzie, 1993), cuja velocidade de 


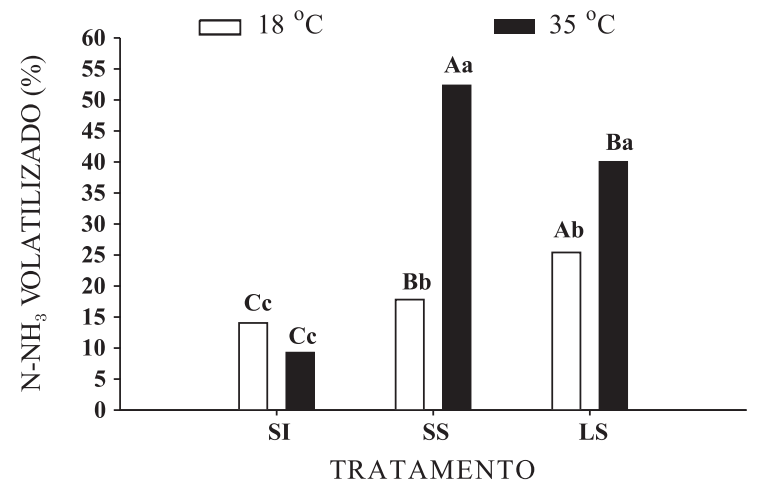

Figura 3. Volatilização de $\mathrm{NH}_{3}$ do solo após a aplicação de ureia convencional ou de ureia com inibidor de urease nas formas sólida incorporada (SI) ou sem incorporação (SS), ou líquida sobre a superfície (LS), em ambiente mantido a 18 ou a $35^{\circ} \mathrm{C}$. Médias das duas fontes de N. Letras maiúsculas comparam as médias dos tratamentos para a mesma temperatura, enquanto as letras minúsculas comparam as médias entre as temperaturas para o mesmo tratamento; letras diferentes demonstram diferenças entre as variáveis analisadas.

reação, que resulta na formação de $\mathrm{NH}_{3}$, aumenta com a diminuição da acidez (Sengik et al., 2001), predispondo o $\mathrm{N}$ a ser perdido para a atmosfera. O potencial de perdas de $\mathrm{NH}_{3}$ diminui quando essa forma de $\mathrm{N}$ transforma-se em amônio, cuja magnitude depende do $\mathrm{pH}$ em torno do grânulo da ureia (Fan \& Mackenzie, 1993) e da umidade do solo (Da Ros et al., 2005). Quanto maior o valor de $\mathrm{pH}$ alcançado, menor é a proporção de $\mathrm{N}^{-} \mathrm{NH}_{4}{ }^{+}$e maior o potencial de volatilização (Rochette et al., 2009b). Os maiores valores de $\mathrm{pH}$ testados proporcionaram as maiores perdas diárias de $\mathrm{NH}_{3}$ - fenômeno também verificado por outros autores (Fan \& Mackenzie, 1993; Sengik et al., 2001).

Os picos de perdas máximas de $\mathrm{NH}_{3}$ ocorreram na primeira semana. As maiores perdas diárias foram observadas entre o quarto e o oitavo dia após a aplicação dos fertilizantes, porém os picos para a ureia convencional aconteceram antes daqueles verificados para a ureia com inibidor de urease, à semelhança do ocorrido no experimento 1 . Além disso, os valores máximos diários de $\mathrm{NH}_{3}$ volatilizada dos tratamentos com ureia foram maiores do que os do solo tratado com ureia com inibidor de urease, fato que se repetiu durante todo o experimento. No quarto dia após a aplicação dos fertilizantes, a ureia volatilizou mais de $1,5 \mathrm{~kg} \mathrm{ha}^{-1} \mathrm{dia}^{-1}$ de $\mathrm{N}$ no solo com $\mathrm{pH} 6,8$, enquanto para a ureia com inibidor de urease o valor ficou abaixo de $1,2 \mathrm{~kg} \mathrm{ha}^{-1} \mathrm{dia}^{-1}$, ambas na temperatura de $18^{\circ} \mathrm{C}$. As perdas diárias de $\mathrm{NH}_{3}$ estabilizaram-se próximas de zero aos 15 dias após a aplicação dos fertilizantes.

As perdas acumuladas totais de $\mathrm{NH}_{3}$ a partir da ureia aplicada na superfície superaram $29 \mathrm{~kg} \mathrm{ha}^{-1}$, enquanto as perdas observadas para a condição da aplicação de ureia com inibidor de urease foram aproximadamente de $23 \mathrm{~kg} \mathrm{ha}^{-1}$. Nos tratamentos com ureia, o efeito do aumento do $\mathrm{pH}$ no incremento da volatilização de $\mathrm{NH}_{3}$ foi maior do que naqueles onde foi aplicada ureia com inibidor de urease. No solo com pH igual a 6,8, a adição de ureia proporcionou perdas de $\mathrm{N}$ próximas a $30 \%$ do total adicionado ao solo. $\mathrm{Na}$ faixa de $\mathrm{pH}$ de 5,5 a 6,3, a volatilização total de $\mathrm{NH}_{3}$ variou entre 17 e $20 \%$ do $\mathrm{N}$ aplicado pela ureia com inibidor de urease e entre 20 e $24 \%$ do N aplicado via ureia convencional.

\section{Experimento 3. Efeito da dose de $\mathrm{N}$ e da forma de aplicação dos fertilizantes}

As perdas diárias e as perdas acumuladas de $\mathrm{NH}_{3}$ a partir da aplicação dos fertilizantes sobre a superfície do solo aumentaram com a dose de $\mathrm{N}$ aplicada (Figura 5), não sendo notada diferença entre as fontes ureia e ureia com inibidor de urease. Na menor dose de $\mathrm{N}\left(100 \mathrm{~kg} \mathrm{ha}^{-1}\right)$, a máxima perda diária desse nutriente foi de $6 \mathrm{~kg} \mathrm{ha}^{-1}$, enquanto na maior dose

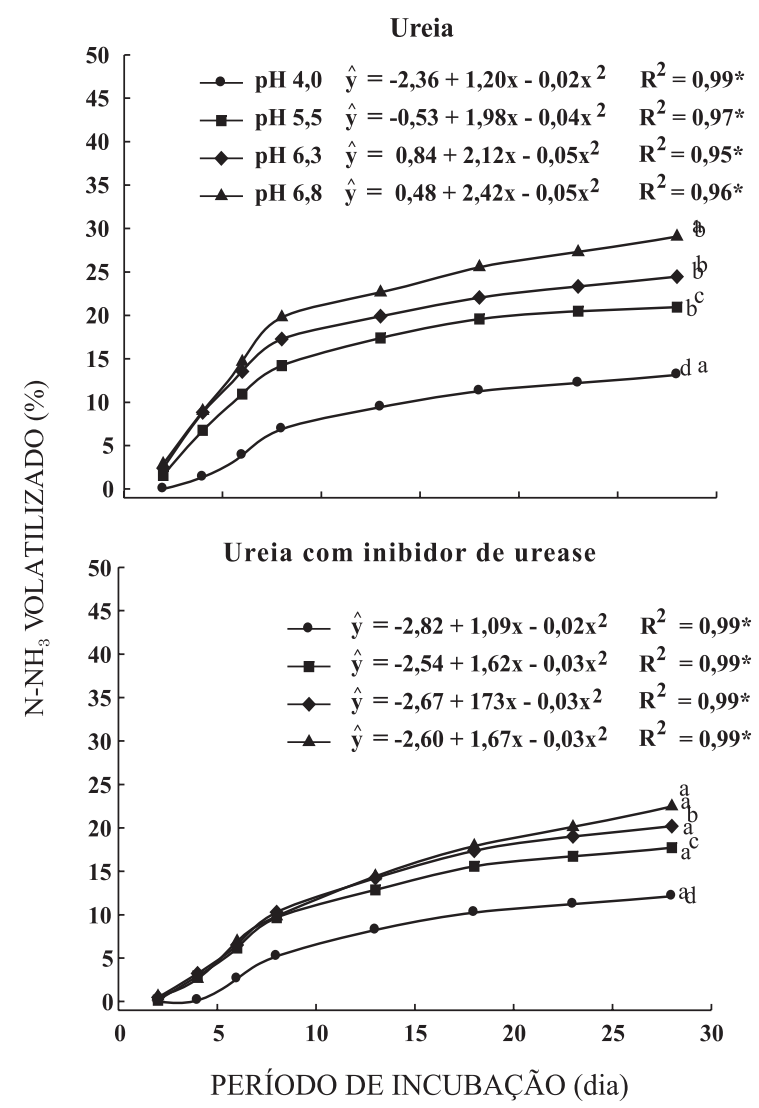

Figura 4. Volatilização acumulada de $\mathrm{NH}_{3}$ do solo ao longo do tempo a partir da aplicação de $100 \mathrm{~kg} \mathrm{ha}^{-1}$ de $\mathrm{N}$ na forma de ureia convencional ou de ureia com inibidor de urease, mantido na temperatura ambiente de $18^{\circ} \mathrm{C}$. Letras diferentes representam diferença estatística $(p<0,05)$ entre as fontes para o mesmo $\mathrm{pH}$, pelo teste de Tukey. * Significativo $(p<0,01)$. 
(190 kg ha-1) a volatilização atingiu 15 e $21 \mathrm{~kg} \mathrm{ha}^{-1}$ dia $^{-1}$ para a aplicação das formas líquida e sólida, respectivamente. As maiores perdas diárias de $\mathrm{NH}_{3}$ ocorreram quatro dias após a implantação do experimento, nas duas doses de $\mathrm{N}$ avaliadas. As perdas acumuladas de $\mathrm{NH}_{3}$ foram três vezes menores para a dose de $100 \mathrm{~kg} \mathrm{ha}^{-1}$ do que para a dose de $190 \mathrm{~kg} \mathrm{ha}^{-1} \mathrm{de}$ $\mathrm{N}$, na média dos dois fertilizantes (ureia e ureia com NBPT) e das duas formas físicas (sólida e líquida) (Figura 5). Na maior dose, quando o fertilizante sólido foi aplicado sobre a superfície do solo, a perda total de $\mathrm{N}$ por volatilização de $\mathrm{NH}_{3}$ ultrapassou o equivalente a $49 \mathrm{~kg} \mathrm{ha}{ }^{-1}$, ou seja, correspondeu a $26,3 \%$ do total de $\mathrm{N}$ adicionado. Apesar de alto, esse valor é inferior aos $78 \%$ encontrados por Lara Cabezas et al. (1997), ao aplicarem ureia sobre a superfície de um Latossolo Vermelho-Escuro muito argiloso cultivado com milho, em Minas Gerais. Scharlau et al. (2007) verificaram que a maior perda de $\mathrm{NH}_{3}(18,5 \%)$ ocorreu a partir da aplicação de ureia no solo que recebeu a maior dose $\left(140 \mathrm{~kg} \mathrm{ha}^{-1} \mathrm{de} \mathrm{N}\right)$, tendo sido maior do que os 7,53\% observados no solo fertilizado com ureia contendo inibidor de urease. A maior perda de $\mathrm{NH}_{3}$ verificada com a aplicação da maior dose de $\mathrm{N}$ também foi observada por Fan \& Mackenzie (1993) e por Ma et al. (2010) e, provavelmente, deve-se ao efeito alcalino na região próxima ao grânulo da ureia (Ernani et al., 2001; Rochette et al., 2009b), o qual ocorre por ocasião da hidrólise da ureia, como consequência da conversão de $\mathrm{N}$ amídico em $\mathrm{NH}_{3}$ (Villas Boas et al., 2005). Ma et al. (2010) propuseram o parcelamento da dose de ureia como alternativa para diminuir a volatilização de $\mathrm{NH}_{3}$.

O efeito do estado físico dos fertilizantes aplicados sobre a superfície do solo na volatilização de $\mathrm{NH}_{3}$ variou com a dose de $\mathrm{N}$ (Figura 5). Na dose de $\mathrm{N}$ equivalente a $190 \mathrm{~kg} \mathrm{ha}^{-1}$, a aplicação na forma líquida promoveu volatilização de $11 \%$ a menos de $\mathrm{NH}_{3}$ do que na forma sólida. As quantidades totais de $\mathrm{NH}_{3}$ volatilizada foram de 26 e $19 \%$ do $\mathrm{N}$ aplicado, respectivamente para as formas líquida e sólida. Quando o fertilizante é aplicado na forma líquida, parte de suas moléculas migra para o interior do solo, além de ocorrer menor incremento no $\mathrm{pH}$ do solo na região adubada, que é menor para a ureia no estado líquido relativamente ao sólido, pois a solução entra em contato com maior número de partículas de solo em relação aos grânulos. Esses dois fenômenos contribuem para a diminuição da volatilização de $\mathrm{NH}_{3}$, em razão da aplicação de fertilizantes amídicos sobre a superfície do solo. $\mathrm{Na}$ menor dose de $\mathrm{N}$, equivalente à adição de $100 \mathrm{~kg} \mathrm{ha}^{-1}$, o estado físico do fertilizante aplicado sobre a superfície do solo (sólido ou líquido) não alterou a perda de $\mathrm{NH}_{3}$.

\section{Experimento 4. Efeito da umidade e do estado físico do fertilizante}

Da mesma forma que o ocorrido nos demais experimentos, as perdas máximas diárias de $\mathrm{NH}_{3}$ ocorreram na primeira semana, ou seja, entre quatro e seis dias após a aplicação da ureia convencional e no sexto dia após a aplicação da ureia com inibidor de urease. A máxima volatilização diária de $\mathrm{N}$ nos tratamentos com ureia foi equivalente a aproximadamente $6 \mathrm{~kg} \mathrm{ha}^{-1}$ dia $^{-1}$ e ocorreu quando o fertilizante foi aplicado no estado sólido no solo com $10 \%$ de umidade. Para a ureia com inibidor de urease, a perda máxima diária de $\mathrm{N}$ foi menor do que $4 \mathrm{~kg} \mathrm{ha}^{-1}$ e ocorreu para o fertilizante aplicado no estado sólido. Depois de transcorridos 10 dias da aplicação dos fertilizantes ao solo, as perdas diárias de $\mathrm{N}-\mathrm{NH}_{3}$ diminuíram para menos de $1 \mathrm{~kg} \mathrm{ha}^{-1}$ dia $^{-1}$, evidenciando que a volatilização de $\mathrm{NH}_{3}$ a partir da aplicação de ureia sobre a superfície do solo ocorre praticamente na primeira semana após a adição do fertilizante (Sangoi et al., 2003).

O estado físico (sólido ou líquido) em que os fertilizantes foram aplicados sobre a superfície do solo influenciou a volatilização de $\mathrm{NH}_{3}$ (Figura 6), à semelhança do ocorrido no experimento 3 . Os dois



Figura 5. Volatilização acumulada de $\mathrm{NH}_{3}$ do solo ao longo do tempo após a aplicação sobre a superfície do solo de doses de $\mathrm{N}\left(\mathrm{a}=100 \mathrm{~kg} \mathrm{ha}^{-1}\right.$; $\mathrm{b}=190 \mathrm{~kg} \mathrm{ha}^{-1}$ ) na forma de ureia convencional ou de ureia com inibidor de urease, no estado sólido (SS) ou líquido (LS). Médias das duas fontes com quatro repetições cada. Letras maiúsculas comparam as médias entre as doses para a mesma forma de aplicação. Letras minúsculas comparam as formas de aplicação para a mesma dose. Letras iguais não diferem entre si a $5 \%(p<0,05)$ para a mesma dose aplicada. * Significativo $(\mathrm{p}<0,01)$. 


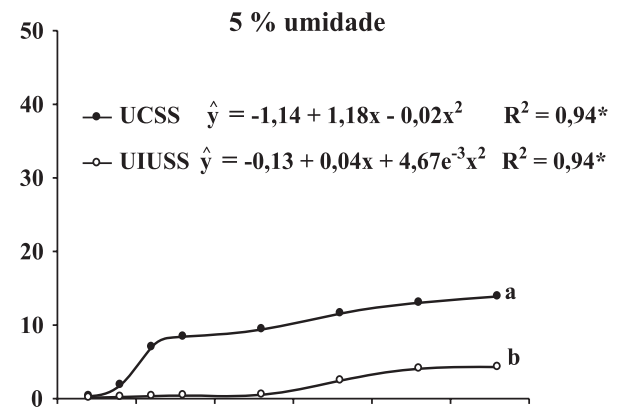

$10 \%$ umidade



$20 \%$ umidade

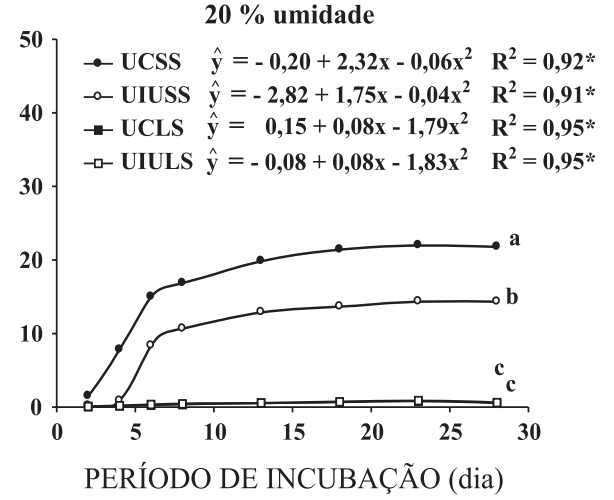

Figura 6. Volatilização acumulada de $\mathrm{NH}_{3}$ ao longo do tempo após a aplicação sobre a superfície do solo de ureia convencional (UC) ou de ureia com inibidor de urease (UIU), no estado sólido (SS) ou líquido (LS), em três teores de água no solo (5, 10 e $20 \%)$. Letras diferentes indicam diferenças entre os tratamentos para a mesma umidade do solo, pelo teste de Tukey $(p<0,05)$. * Significativo $(\mathrm{p}<0,01)$.

fertilizantes (ureia convencional e ureia com inibidor de urease) proporcionaram menor perda de $\mathrm{NH}_{3}$, tanto diária quanto acumulada, quando foram aplicados na forma líquida. Quando aplicados no estado líquido, as perdas totais acumuladas durante todo o período experimental foram inferiores a $2 \mathrm{~kg} \mathrm{ha}^{-1}$ de $\mathrm{N}$, independentemente da presença ou não de inibidor da urease (NBPT) no fertilizante e do conteúdo de água do solo (Figura 6). O teor de água do solo é um fator importante na hidrólise da ureia: quando o solo está seco, a atividade da urease é baixa (Rochette et al., 2009b); com o incremento da umidade no solo, a atividade enzimática aumenta, pois a adição de água promove o aumento da difusão da ureia e, consequentemente, maior contato com a urease existente no solo (Savant et al., 1987).

As fontes de N (ureia convencional e ureia com inibidor de urease) promoveram volatilização na forma de $\mathrm{NH}_{3}$ de maneira diferente com a variação da umidade do solo (Figura 6). As perdas totais de $\mathrm{NH}_{3}$ diferiram entre os dois fertilizantes quando eles foram aplicados sobre a superfície do solo, porém somente para aqueles adicionados no estado sólido. Independentemente da umidade $(p<0,05)$ no solo, a volatilização foi maior para a ureia convencional do que para a ureia com NBPT. O tratamento com $10 \%$ de umidade foi o que proporcionou a maior emissão de $\mathrm{NH}_{3}$, tendo atingido o equivalente a 28 e $15 \mathrm{~kg} \mathrm{ha}^{-1}$, respectivamente, nas parcelas em que foram aplicadas a ureia convencional e a ureia com inibidor de urease.

O teor de água do solo influenciou a volatilização de $\mathrm{NH}_{3}$ (Figura 6), de modo que as menores volatilizações ocorreram no solo seco - fenômeno também observado por Duarte et al. (2007), provavelmente em razão da diminuição da hidrólise da ureia (Rochette et al., 2009b), uma vez que a atividade da urease é baixa nessas condições (Cantarella et al., 2003). A aplicação de ureia em solo seco acarretou perda semelhante à do tratamento sem aplicação de ureia, o que indica ausência de volatilização de $\mathrm{N}$ durante o período de avaliação com o solo nessas condições (Duarte et al., 2007).

As perdas de $\mathrm{NH}_{3}$ obtidas neste trabalho podem subestimar as perdas que ocorrem no campo, uma vez que, em laboratório, as condições são menos propícias à volatilização, principalmente pela ausência de vento e alterações na luminosidade e nos processos de umedecimento e secagem do solo. Araújo et al. (2009) verificaram, em laboratório, que a volatilização de $\mathrm{NH}_{3}$ foi de apenas $57 \%$ daquela ocorrida no campo, ao utilizarem fertilizante nitrogenado isotopicamente marcado $\left({ }^{15} \mathrm{~N}\right)$ e método de captura do $\mathrm{N}$ volatilizado similar ao empregado no presente estudo.

\section{CONCLUSÕES}

1. A incorporação ao solo tanto da ureia convencional como da ureia com inibidor de urease é a melhor forma de prevenir a volatilização de $\mathrm{NH}_{3}$. $\mathrm{Na}$ maioria das circunstâncias, a aplicação desses fertilizantes no estado líquido também promove menor perda de $\mathrm{NH}_{3}$ do que a aplicação no estado sólido.

2. A volatilização de $\mathrm{NH}_{3}$ aumenta com o acréscimo da dose de $\mathrm{N}$ aplicada, do $\mathrm{pH}$ do solo e da temperatura, e foi maior quando o solo apresentou teor intermediário de umidade, em comparação aos extremos.

3. Em relação à ureia convencional, a ureia com inibidor de urease retarda os picos de volatilização de $\mathrm{NH}_{3}$, os quais aconteceram sempre na primeira semana após a aplicação dos fertilizantes sobre a 
superfície do solo, porém nem sempre proporciona menor perda quantitativa de $\mathrm{N}$.

4. A perda máxima acumulada de $\mathrm{NH}_{3}$ chegou a atingir $50 \%$ do $\mathrm{N}$ aplicado, nas condições mais favoráveis à volatilização, ou seja, quando os fertilizantes foram aplicados sobre a superfície do solo e a temperatura do ambiente foi mantida em $35^{\circ} \mathrm{C}$.

\section{LITERATURA CITADA}

AL-KANANI, T.; MACKENZIE, A.F. \& BARTHAKUR, N.N. Soil water and ammonia volatilization relationships with surface-applied nitrogen fertilizer solutions. Soil Sci. Soc. Am. J., 55:1761-1766, 1991.

ARAÚJO, E.S.; MARSOLA, T.; MIYAZAWA, M.; SOARES, L.H.B.; URQUIAGA, S.; BODDEY, R.M. \& ALVES, B.J.R. Calibração de câmara semiaberta estática para quantificação de amônia volatilizada do solo. Pesq. Agropec. Bras., 44:769-776, 2009.

CANTARELLA, H.; MATTOS Jr., D.; QUAGGIO, J.A. \& RIGOLIN, A.T. Fruit yield of Valencia sweet orange fertilized with different $\mathrm{N}$ sources and the loss of applied N. Nutr. Cycling Agroecosyst., 67:215-223, 2003.

CANTARELLA, H. \& MARCELINO, R. O uso de inibidor de urease para aumentar a eficiência da ureia. In: SIMPOSIO SOBRE INFORMAÇÕES RECENTES PARA OTIMIZAÇÃO DA PRODUÇÃO AGRÍCOLA, 2007, Piracicaba. Anais... Piracicaba, 2007.v.1. p.2-19

CANTARELLA, H.; TRIVELIM, P.C.O.; CONTIN, T.L.M.; DIAS, F.L.F.; ROSSETTO, R.; MARCELINO, R.; COIMBRA, R.B. \& QUAGGIO, J.A. Ammonia volatilisation from urease inhibitor-treated urea applied to sugarcane trash blankets. Sci. Agric., 65:397-401, 2008.

CLAY, D.E.; MALZER, G.L. \& ANDERSON, J.L. Ammonia volatilization from urea as influenced by soil temperature, soil water content, and nitrification and hydrolysis inhibitors. Soil Sci. Soc. Am. J., 54:263-266, 1990.

DA ROS, C.O.; AITA, C. \& GIACOMINI, S.J. Volatilização de amônia com aplicação de ureia na superfície do solo, no sistema plantio direto. Ci. Rural, 35:799-805, 2005.

DUARTE, F.M.; POCOJESKI, E.; SILVA, L.S.; GRAUPE, F.A. \& BRITZKE, D. Perdas de nitrogênio por volatilização de amônia com aplicação de ureia em solo de várzea com diferentes níveis de umidade. Ci. Rural, 37:705-711, 2007.

EMPRESA BRASILEIRA DE PESQUISA AGROPECUÁRIA . EMBRAPA. Centro Nacional de Pesquisa de Solos. Sistema brasileiro de classificação de solos. Rio de Janeiro, 1999. $412 \mathrm{p}$

ERNANI, P.R.; BAYER, C. \& STECKLING, C. Características químicas de solo e rendimento de matéria seca de milho em função do método de aplicação de fosfatos, em dois níveis de acidez. R. Bras. Ci. Solo, 25:939-946, 2001.

ERNEST, J.W. \& MASSEY, H.F. The effects of several factors on volatilization of ammonia formed from urea in the soil. Soil Sci. Soc. Am. Proc., 24:87-90, 1960.
FAN, M.X. \& MACKENZIE, A.F. Urea and phosphate interactions in fertilizer microsites: Ammonia volatilization and ph changes. Soil Sci. Soc. Am. J., 57:839845,1993

LARA-CABEZAS, W.A.R.; KORNDORFER, G. \& MOTTA, S.A. Volatilização de $\mathrm{N}^{-\mathrm{NH}_{3}}$ na cultura de milho: I. Efeito da irrigação e substituição parcial da ureia por sulfato de amônio. R. Bras. Ci. Solo, 21:481-487, 1997.

LARA-CABEZAS, W.A.R.; TREIVELIN, P.C.O.; KORNDORFER, G. \& PEREIRA, G.H. Balanço da adubação nitrogenada sólida e fluída de cobertura na cultura de milho, em sistema de plantio direto no triangulo mineiro. R. Bras. Ci. Solo, 24:363-476, 2000.

LIU, G.; LI, Y. \& ALVA, A.K. High water regime can reduce ammonia volatilization from soils under potato production. Comm. Soil Sci. Plant Anal., 38:1203-1220, 2007.

MA, B.L.; WU, T.Y.; TREMBLAY, N.; DEEN, W.; MCLAUGHLIN, N.B.; MORRISON M. J. \& STEWART, G. On-farm assessment of the amount and timing of nitrogen fertilizer on ammonia volatilization. Agron. J., 102:134-144, 2010.

MIYAZAWA, M. Método de captação da amônia volatilizada do solo. Londrina, IAPAR, Instituto Agronômico do Paraná, 2007. 2 p.

MOYO, C.C.; KISSEL, D. E. \& CAB4RERA, M. L. Temperature effects on soil urease activity. Soil Biol. Biochem., 21:935938, 1989

O'CONNOR, M.J. \& HENDRICKSON, L.L. Effect of phenylphosphorodiamidate on ammonia volatilization as affected by soil temperature and rates and distribution of urea. Soil Sci. Soc. Am. J., 51:1062-1066, 1987.

ROCHETTE, P.; ANGERS, D.; CHANTINI, M.H.; MacDONALD, J.D.; GASSER, M. \& BERTRAND, N. Reducing ammonia volatilization in a no-till soil by incorporating urea and pig slurry in shallow bands. Nutr. Cycling Agroecosyst., 84:71-80, 2009a.

ROCHETTE, P.; MacDONALD, J.D.; ANGERS, D.; CHANTINI, M.H.; GASSER, M. \& BERTRAND, N. Banding urea increased ammonia volatilization in a dry acidic soil. J. Environ. Qual., 38:1383-1390, 2009b.

ROCHETTE, P.; ANGERS, D.; CHANTINI, M.H.; MacDONALD, J.D.; BISSONNETTE, N. \& BERTRAND, $\mathrm{N}$. Ammonia volatilization following surface application of ura to tilled and no-till soils: A laboratory comparison. Soil Tillage Res., 103:310-315, 2009c.

RODRIGUES, M.B. \& KIEHL, J.C. Volatilização de amônia após emprego de ureia em diferentes doses e modos de aplicação. R. Bras. Ci. Solo, 10:37-43, 1986.

SANGOI, L.; ERNANI, P.R.; LECH, V.A. \& RAMPAZZO, C. Volatilização de $\mathrm{N}-\mathrm{NH}_{3}$ em decorrência da forma de aplicação de ureia, manejo de resíduos e tipo de solo, em laboratório. Ci. Rural, 33:87-692, 2003. 
SCHARLAU, A.V.; ESCOSTEGUY, P.A.V.; RIVA, E. \& PASCOALOTTO, M. Volatilização de amonia em solo com uréia, super $\mathrm{N}$ e nitrato de amônio. In: CONGRESSO BRASILEIRO DE CIÊNCIA DO SOLO, 31, 2007. Anais. Gramado. Sociedade Brasileira de Ciência do Solo, 2007. CD-ROM.

SANZ-COBENA, A.; MISSELBROOK, T.H.; ARCE, A.; MINGOT, J.I.; DIEZ, J.A. \& VALLEJO, A. An inhibitor of urease activity effectively reduces ammonia emissions from soil treated with urea under Mediterranean conditions. Agric. Ecosyst. Environ., 126:243-249, 2008.

SAVANT, N.K.; JAMES, A.F. \& MCCLELLAN, G.H. Effect of amounts and sequence of additions of urea and water on hydrolysis of surface-applied granular urea in unsaturated soils. Fert. Res., 11:231-234, 1987.

SENGIK, E.; KIEHL, J.C. \& SILVA, M.A.G. Perdas de amônia em solo e de resíduos orgânicos autoclavados e tratados com ureia. Acta Sci., 23:1099-1105, 2001.
SENGIK, E. \& KIEHL, J.C. Efeito de resíduos orgânicos e do fosfato monocálcico na volatilização de amônia em terra tratada com ureia. R. Bras. Ci. Solo, 19:321-326, 1995a.

TEDESCO, M.J.; GIANELLO, C.; BISSANI, C.A. \& BOHNEN, H. Análise de solo, plantas e outros materiais. 2.ed. Porto Alegre, Universidade Federal do Rio Grande do Sul, 1995. 147p. (Boletim Técnico, 5)

TRIVELIN, P.C.O.; OLIVEIRA, M.W.; CESAR, V.A.; GAVA, G.J.C. \& BENDASSOLLI, J.A. Perdas do nitrogênio da ureia no sistema solo-planta em dois ciclos de cana-deaçúcar. Pesq. Agropec. Bras., 37:193-201, 2002.

VILLAS BOAS, R.L.; BOARETTO, A.E. \& GODOY, L.J.G. Nitrogen recovery of urea - ammonium sulphate mixtures by corn plants. Bragantia, 64:263-272, 2005. 